\title{
An Equiconsistency for Universal Indestructibility *††
}

\author{
Arthur W. Apter ${ }^{\S}$ \\ Department of Mathematics \\ Baruch College of CUNY \\ New York, New York 10010 USA \\ and \\ The CUNY Graduate Center, Mathematics \\ 365 Fifth Avenue \\ New York, New York 10016 USA \\ http://faculty.baruch.cuny.edu/apter \\ awapter@alum.mit.edu \\ Grigor Sargsyan \\ Group in Logic and the Methodology of Science \\ University of California \\ Berkeley, California 94720 USA \\ http://math.berkeley.edu/ grigor \\ grigor@math.berkeley.edu \\ June 30, 2008 \\ (revised March 6, 2009)
}

\begin{abstract}
We obtain an equiconsistency for a weak form of universal indestructibility for strongness. The equiconsistency is relative to a cardinal weaker in consistency strength than a Woodin cardinal, Stewart Baldwin's notion of hyperstrong cardinal. We also briefly indicate how our methods are applicable to universal indestructibility for supercompactness and strong compactness.
\end{abstract}

*2000 Mathematics Subject Classifications: 03E35, 03E45, 03E55.

${ }^{\dagger}$ Keywords: Universal indestructibility, indestructibility, equiconsistency, measurable cardinal, strong cardinal, hyperstrong cardinal, Woodin cardinal, strongly compact cardinal, supercompact cardinal, core model.

¥The authors owe a huge debt of gratitude to both Joel Hamkins and Ralf Schindler. Without their help, encouragement, and many conversations on the subject matter contained herein, this paper would not have been possible. The authors also wish to thank the referee, for helpful comments, suggestions, and corrections which have been incorporated into the current version of the paper.

$\S$ The first author's research was partially supported by PSC-CUNY grants and CUNY Collaborative Incentive grants. 
Ever since the first author and Hamkins introduced the concept of universal indestructibility in [1], every theorem proven has used large cardinals whose consistency strength is beyond supercompactness. The reason for this is that indestructibility of whatever property the cardinal $\kappa$ possesses (measurability, full or partial versions of strongness, strong compactness, supercompactness, etc.) has been under all $<\kappa$-directed closed partial orderings ${ }^{1}$, including the collapse forcings $\operatorname{Coll}(\kappa, \lambda)$ and $\operatorname{Coll}(\kappa,<\lambda)$ for $\lambda>\kappa$ an arbitrary cardinal. Thus, core model considerations (see, e.g., [10] and [11]) indicate the necessity of very large cardinals.

The purpose of this paper is to show that by weakening what we consider, it is possible to reduce the consistency strength used to establish our form of universal indestructibility below supercompactness and actually obtain an equiconsistency. Before stating our main theorem, however, we briefly digress to give the relevant terminology and definitions. We begin with a definition due to Stewart Baldwin [3], that of a hyperstrong cardinal $\kappa .^{2}$

Definition 1.1 (S. Baldwin) $\kappa$ is 0 hyperstrong iff $\kappa$ is strong. $\kappa$ is $\alpha+1$ hyperstrong iff for any ordinal $\delta>\kappa$, there is an elementary embedding $j: V \rightarrow M$ witnessing the $\delta$ strongness of $\kappa$ (i.e., $c p(j)=\kappa, j(\kappa)>\left|V_{\delta}\right|$, and $\left.V_{\delta} \subseteq M\right)$ generated by a $(\kappa, \delta)$-extender such that $M \vDash " \kappa$ is $\alpha$ hyperstrong". For $\lambda$ a limit ordinal, $\kappa$ is $\lambda$ hyperstrong iff for any ordinal $\delta>\kappa$, there is an elementary embedding $j: V \rightarrow M$ witnessing the $\delta$ strongness of $\kappa$ generated by a $(\kappa, \delta)$-extender such that $M \vDash " \kappa$ is $\alpha$ hyperstrong for every $\alpha<\lambda$ ". $\kappa$ is hyperstrong iff $\kappa$ is a hyperstrong for every ordinal $\alpha$.

Definition 1.1 easily implies that a hyperstrong cardinal is quite large in size. In particular, a hyperstrong cardinal is a limit of strong cardinals.

When forcing, $q \geq p$ means that $q$ is stronger than $p$. If $\mathbb{P} \in V$ is a partial ordering and $G \subseteq V$ is $V$-generic over $\mathbb{P}$, then we will abuse notation somewhat and use $V[G]$ and $V^{\mathbb{P}}$ interchangeably to denote the generic extension. If $\mathbb{P}$ is a partial ordering and $\kappa$ is a cardinal, $\mathbb{P}$ is $\kappa$-strategically closed if in the two person game in which the players construct an increasing sequence $\left\langle p_{\alpha}: \alpha \leq \kappa\right\rangle$, where

\footnotetext{
${ }^{1}$ In Laver's original paper [9], these partial orderings were referred to as being $\kappa$-directed closed.

${ }^{2}$ Although our definition differs slightly from the one found in [3], the two are equivalent.
} 
player I plays odd stages and player II plays even stages (choosing the trivial condition at stage 0 ), player II has a strategy which ensures the game can always be continued. $\mathbb{P}$ is $\prec \kappa$-strategically closed if in the two person game in which the players construct an increasing sequence $\left\langle p_{\alpha}: \alpha<\kappa\right\rangle$, where player I plays odd stages and player II plays even stages (choosing the trivial condition at stage 0), player II has a strategy which ensures the game can always be continued. $\mathbb{P}$ is $<\kappa$ strategically closed if $\mathbb{P}$ is $\delta$-strategically closed for every cardinal $\delta<\kappa$. $\mathbb{P}$ is $(\kappa, \infty)$-distributive if for every sequence $\left\langle D_{\alpha}: \alpha<\kappa\right\rangle$ of dense open subsets of $\mathbb{P}, \bigcap_{\alpha<\kappa} D_{\alpha}$ is also a dense open subset of $\mathbb{P}$. Note that since forcing with a partial ordering which is $(\kappa, \infty)$-distributive adds no new subsets of $\kappa$, the measurability of any measurable cardinal $\kappa$ (or equivalently, its $\kappa+1$ strongness) is automatically indestructible under such partial orderings.

As in [7], if $\mathcal{A}$ is a collection of partial orderings, then the lottery sum is the partial ordering $\oplus \mathcal{A}=\{\langle\mathbb{P}, p\rangle: \mathbb{P} \in \mathcal{A}$ and $p \in \mathbb{P}\} \cup\{0\}$, ordered with 0 below everything and $\langle\mathbb{P}, p\rangle \leq\left\langle\mathbb{P}^{\prime}, p^{\prime}\right\rangle$ iff $\mathbb{P}=\mathbb{P}^{\prime}$ and $p \leq p^{\prime}$. Intuitively, if $G$ is $V$-generic over $\oplus \mathcal{A}$, then $G$ first selects an element of $\mathcal{A}$ (or as Hamkins says in [7], "holds a lottery among the posets in $\mathcal{A}$ ") and then forces with it. The terminology "lottery sum" is due to Hamkins, although the concept of the lottery sum of partial orderings has been around for quite some time and has been referred to at different junctures via the expressions "disjoint sum of partial orderings," "side-by-side forcing," and "choosing which partial ordering to force with generically."

Recall that universal indestructibility for supercompactness holds in a model $V$ for ZFC if every $V$-supercompact and partially supercompact (including measurable) cardinal $\delta$ has its degree of supercompactness fully Laver indestructible [9] under $<\delta$-directed closed forcing. There are exactly analogous definitions of universal indestructibility for measurability, strongness, or strong compactness, for which we refer readers to [1] for further details. This motivates the definition that weak universal indestructibility for strongness holds in a model $V$ for ZFC if every $V$-strong and partially strong (including measurable) cardinal $\delta$ has its degree of strongness indestructible under partial orderings which are both $<\delta$-strategically closed and $(\delta, \infty)$-distributive. If some measurable cardinal $\delta$ has its degree of strongness indestructible under partial orderings which are 
both $<\delta$-strategically closed and $(\delta, \infty)$-distributive, then $\delta$ 's degree of strongness will be said to be weakly indestructible. In addition, a partial ordering $\mathbb{P}$ which is both $<\delta$-strategically closed and $(\delta, \infty)$-distributive will be said to be $\delta$-suitable.

We are now able to state our main theorem.

Theorem 1 Weak universal indestructibility for strongness in the presence of a strong cardinal is equiconsistent with a hyperstrong cardinal.

Some discussion of the genesis of this paper is perhaps in order here. Apter had the idea that the form of universal indestructibility discussed might generate an equiconsistency. He proposed to Sargsyan that this equiconsistency be established using a Woodin cardinal, which by the work of our earlier paper [2], is sufficient for the forcing portion of Theorem 1. Email correspondence with Schindler then indicated that weak universal indestructibility was unlikely to yield an inner model containing a Woodin cardinal. Over a period of several months, intense discussions (both in person and by email) ensued among Apter, Hamkins, Sargsyan, and Schindler. Attempts were made to demonstrate the equiconsistency by using two cardinals $\kappa_{1}<\kappa_{2}$ such that $\kappa_{1}$ had strongness properties and $\kappa_{2}$ had $\Sigma_{2}$ reflection properties. This approach, however, was misleading in our efforts to prove Theorem 1. In particular, it could not result in an equiconsistency, because the forcing portion of the proof required only a limited amount of $\Sigma_{2}$ reflection. These observations forced us to reconsider the consistency proofs for universal indestructibility, which led Sargsyan to coming up with the key techniques used in the current proof of Theorem 1 . This approach in turn led to the definition of a large cardinal, which we initially called an enhanced strong cardinal, that didn't have the ad hoc $\Sigma_{2}$ reflection properties but could still be used for the forcing part of our proof. Later it came to our attention that S. Baldwin had already studied this notion in [3], which prompted us to adopt his notation and terminology for this paper.

Before turning our attention to the proof of Theorem 1, we note that a hyperstrong cardinal has consistency strength strictly weaker than a Woodin cardinal. More explicitly, we have the following theorem. 
Theorem $2 Z F C+$ There is a Woodin cardinal $\vdash$ Con $(Z F C+$ There is a proper class of hyperstrong cardinals).

Proof: Suppose $\kappa$ is a Woodin cardinal. Assume towards a contradiction that $\delta<\kappa$ is such that $V_{\kappa} \vDash$ "No cardinal $\lambda>\delta$ is a hyperstrong cardinal". Let $f: \kappa \rightarrow \kappa$ be defined by

$$
f(\alpha)=\text { The least } \beta \text { such that } \mathrm{V}_{\kappa} \vDash \text { " } \alpha \text { is not } \beta \text { hyperstrong". }
$$

Let $A$ code the graph of $f$. Because $\kappa$ is a Woodin cardinal, by [8, Theorem 26.14], for any $A \subseteq V_{\kappa}$, there is a proper class of $A$-strong cardinals in $V_{\kappa}$. There thus must be some $\lambda>\delta, \lambda<\kappa$ such that for any $\eta>\lambda, \eta<\kappa$, there is an elementary embedding $j_{\eta}: V \rightarrow M_{\eta}$ witnessing the $\eta$ strongness of $\lambda$ generated by a $(\lambda, \eta)$-extender $\mathcal{E}_{\eta} \in V_{\kappa}$ with $A \cap V_{\eta}=j_{\eta}(A) \cap V_{\eta}$. If $\sigma \in A$ codes the pair $\langle\lambda, f(\lambda)\rangle$, then for any $\eta>\max (f(\lambda), \sigma), \eta<\kappa$, we have that $j_{\eta}(f)(\lambda)=f(\lambda)$. As a consequence, in $V_{\kappa}$, for all sufficiently large $\eta<\kappa$, the elementary embedding $k_{\eta}: V \rightarrow M_{\eta}^{*}$ generated by $\mathcal{E}_{\eta}$ is such that $M_{\eta}^{*} \vDash$ " $\lambda$ is $\beta$ hyperstrong for every $\beta<f(\lambda)$ ", i.e., $V_{\kappa} \vDash$ " $\lambda$ is $f(\lambda)$ hyperstrong". This contradiction means we may therefore conclude that $V_{\kappa} \vDash$ "There is a proper class of hyperstrong cardinals". This completes the proof of Theorem 2 .

We turn our attention now to the proof of Theorem 1. For clarity of exposition, we split its presentation into two distinct components. We begin with our forcing construction, i.e., we first prove the following result.

Theorem 3 Con $(Z F C+$ There is a hyperstrong cardinal $) \Longrightarrow$ Con $(Z F C+$ There is a strong cardinal + Weak universal indestructibility for strongness holds).

Proof: Suppose $V \vDash$ "ZFC $+\kappa$ is a hyperstrong cardinal". By [3, Theorem 3.12], it is also possible to assume that $V \vDash \mathrm{GCH}$. Without loss of generality, by truncating the universe if necessary, we assume in addition that $V \vDash$ "No cardinal $\lambda>\kappa$ is $(\lambda+2)$ strong".

Our partial ordering is a modification of the one found in the proof of [1, Theorem 5]. Specifically, we define a reverse Easton iteration having length at most $\kappa$ which begins by adding a Cohen 
subset of $\omega$. All other nontrivial stages of forcing can only occur at a $\delta<\kappa$ which is a Mahlo cardinal in $V$. Suppose $\mathbb{P}_{\delta}$ has been defined. If $\delta$ is not $\delta+2$ strong in $V^{\mathbb{P}_{\delta}}$, then we perform trivial forcing. Otherwise, if there is some condition $p \in \mathbb{P}_{\delta}$ forcing that $\delta$ is $\lambda$ strong for every $\lambda<\eta$, where $\eta$ is the least cardinal in $V^{\mathbb{P}_{\delta}}$ greater than $\delta$ which is $(\eta+2)$ strong, and these degrees of strongness are weakly indestructible via any partial ordering having rank less than $\kappa$, then we stop our construction and take $\left(V_{\eta}\right)^{V^{\mathbb{P}} \delta}$ as our final desired model (assuming we have forced above $p$ ). Finally, if the preceding two cases do not hold, then in $V^{\mathbb{P}_{\delta}}, \delta$ is (at least) $\delta+2$ strong, and there is some minimal $\alpha<\eta, \alpha \geq \delta+2$ such that the $\alpha$ strongness of $\delta$ is destroyed by some $\delta$-suitable partial ordering $\mathbb{Q}^{*}$ of rank less than $\kappa$. By the proof of $[1$, Lemma 4$]$, we may assume that forcing with $\mathbb{Q}^{*}$ leaves no cardinals $\beta$ which are $\beta+2$ strong in the half-open interval $\left(\alpha,\left|\mathbb{Q}^{*}\right|\right]{ }^{3}$ We then let $\mathbb{P}_{\delta+1}=\mathbb{P}_{\delta} * \dot{\mathbb{Q}}_{\delta}$, where $\dot{\mathbb{Q}}_{\delta}$ is a term for the lottery sum of all $\delta$-suitable partial orderings $\mathbb{Q}^{*}$ having rank below the least $V$-strong cardinal above $\delta$ such that forcing with $\mathbb{Q}^{*}$ destroys the $\alpha$ strongness of $\delta$ and leaves no cardinals $\beta$ which are $\beta+2$ strong in the half-open interval $\left(\alpha,\left|\mathbb{Q}^{*}\right|\right]$. The proof of Theorem 3 is now completed by the following three lemmas.

Lemma 1.2 Suppose that $\delta$ and $\eta$ are as in the last case given above in the inductive definition of $\mathbb{P}$, and $\rho<\eta$ is such that $V^{\mathbb{P}_{\delta}} \vDash " \delta$ is $\rho$ strong, and there is a $\delta$-suitable partial ordering $\mathbb{Q}^{* *}$ such that forcing with $\mathbb{Q}^{* *}$ destroys the $\rho$ strongness of $\delta$ and leaves no cardinals $\beta$ which are $\beta+2$ strong in the half-open interval $\left(\rho,\left|\mathbb{Q}^{* *}\right|\right]$ ". Then there is always a $\delta$-suitable partial ordering $\mathbb{Q}^{*} \in V^{\mathbb{P}_{\delta}}$ having rank less than the least $V$-strong cardinal $\gamma$ greater than $\delta$ such that forcing with $\mathbb{Q}^{*}$ destroys the $\rho$ strongness of $\delta$ and leaves no cardinals $\beta$ which are $\beta+2$ strong in the half-open interval $\left(\rho,\left|\mathbb{Q}^{*}\right|\right]$.

Proof: Suppose there is such a partial ordering $\mathbb{Q}^{* *}$ having rank greater than $\gamma$. Let $\lambda>\gamma$ be sufficiently large so that if $j: V \rightarrow M$ is an elementary embedding witnessing the $\lambda$ strongness of $\gamma$, then $M \vDash$ "Forcing with the $\delta$-suitable partial ordering $\mathbb{Q}^{* *}$ over $M^{\mathbb{P}_{\delta}}$ destroys the $\rho$ strongness of $\delta$ and leaves no cardinals $\beta$ which are $\beta+2$ strong in the half-open interval $\left(\rho,\left|\mathbb{Q}^{* *}\right|\right]$, where

\footnotetext{
${ }^{3}$ If necessary, instead of using $\mathbb{Q}^{*}$ alone, force with, e.g., $\mathbb{Q}^{*} * \operatorname{Coll}\left(\lambda,\left|\mathbb{Q}^{*}\right|\right)$, where $\vdash_{\mathbb{Q}^{*}}$ " $\lambda$ is the least inaccessible cardinal greater than $\alpha "$.
} 
$\rho<\eta$ and $\eta$ is the least cardinal greater than $\delta$ which is $(\eta+2)$ strong in $M^{\mathbb{P}} \delta$ ". Since the critical point of $j$ is $\gamma$ and $\delta, \rho, \eta<\gamma$, by reflection, there is a $\delta$-suitable partial ordering $\mathbb{Q}^{*} \in V^{\mathbb{P}_{\delta}}$ having rank less than $\gamma$ such that $V \vDash$ "Forcing with the $\delta$-suitable partial ordering $\mathbb{Q}^{*}$ over $V^{\mathbb{P}_{\delta}}$ destroys the $\rho$ strongness of $\delta$ and leaves no cardinals $\beta$ which are $\beta+2$ strong in the half-open interval $\left(\rho,\left|\mathbb{Q}^{*}\right|\right]$, where $\rho<\eta$ and $\eta$ is the least cardinal greater than $\delta$ which is $(\eta+2)$ strong in $V^{\mathbb{P}_{\delta}}$. This completes the proof of Lemma 1.2.

Lemma 1.2 allows us to infer that if $\delta \leq \kappa$ is a strong cardinal, then $\mathbb{P}_{\delta} \subseteq V_{\delta}$.

Lemma 1.3 If the construction of $\mathbb{P}$ terminates at some stage $\sigma<\kappa$, then $\left(V_{\eta}\right)^{V^{\mathbb{P}} \sigma}$ as described above is a model for weak universal indestructibility for strongness containing a strong cardinal.

Proof: We assume throughout the course of the proof of Lemma 1.3 that we are forcing above the appropriate condition $p$. By the inductive definition of $\mathbb{P}$, the same proof as given for $[1$, Theorem 5$]$ yields that weak indestructibility for strongness must hold in $\left(V_{\eta}\right)^{V^{\mathbb{P}} \sigma}$ for any measurable cardinal $\delta \in\left(V_{\eta}\right)^{V^{\mathbb{P} \sigma}}$ such that $\delta \leq \sigma$ and $V^{\mathbb{P}_{\delta}} \vDash " \delta$ is (at least) $\delta+2$ strong". 4 The only problematic measurable cardinals below $\sigma$ are hence those measurable cardinals $\delta \in\left(V_{\eta}\right)^{V^{\mathbb{P} \sigma}}$ such that $V^{\mathbb{P}_{\delta}} \vDash " \delta$ isn't $\delta+2$ strong". However, by the definition of weak indestructibility, $V^{\mathbb{P}_{\delta}} \vDash$ " $\delta$ 's degree of strongness, namely measurability, is weakly indestructible". Since nontrivial stages of forcing above $\delta$ can only occur at ordinals $\gamma$ such that $V^{\mathbb{P}_{\gamma}} \vDash$ " $\gamma$ is (at least) $\gamma+2$ strong", for $\dot{\mathbb{P}}^{*}$ such that $\mathbb{P}_{\delta} * \dot{\mathbb{P}}^{*}=\mathbb{P}_{\sigma}$, it is the case that $\Vdash_{\mathbb{P}_{\delta}}$ "Forcing with $\dot{\mathbb{P}}^{*}$ adds no new subsets of the least strong limit cardinal above $\delta$ ". Thus, $V^{\mathbb{P}_{\delta} \dot{\mathbb{P}}^{*}}=V^{\mathbb{P}_{\sigma}} \vDash$ " $\delta$ 's degree of strongness, namely measurability, is weakly indestructible", i.e., weak indestructibility for strongness holds in $\left(V_{\eta}\right)^{V^{\mathbb{P}} \sigma}$

\footnotetext{
${ }^{4}$ To see in greater detail why this is the case, we quote almost verbatim from the proof of [1, Theorem 5], making the minor necessary modifications where necessary. Let $\alpha \geq \delta+2, \alpha<\eta$ be the least ordinal such that $V^{\mathbb{P}_{\delta+1}} \vDash$ " $\delta$ isn't $\alpha$ strong". Suppose $V^{\mathbb{P}_{\delta+1}} \vDash$ " $\delta$ is $\beta$ strong". Necessarily, $\beta<\alpha$. In addition, no $\delta$-suitable forcing $\mathbb{Q}^{* *} \in V^{\mathbb{P} \delta+1}$ can destroy the $\beta$ strongness of $\delta$. This is since if $\mathbb{Q}^{*}$ is the forcing chosen by the lottery at stage $\delta$, then $\mathbb{Q}^{*} * \dot{\mathbb{Q}}^{* *}$ would have destroyed the $\beta$ strongness of $\delta$ over $V^{\mathbb{P}_{\delta}}$, contradicting the minimality of $\alpha$. In particular, the $\beta$ strongness of $\delta$ is preserved by the forcing $\mathbb{P}_{\delta, \gamma}$ which leads to any of the later models $V^{\mathbb{P}_{\gamma}}$ for $\gamma<\kappa$. Furthermore, since the next nontrivial stage of forcing after $\delta$ takes place at an inaccessible cardinal beyond $\alpha$ and $\left|\mathbb{Q}^{*}\right|$, the $\alpha$ strongness of $\delta$ is never resurrected by the later stages of forcing. Consequently, in all of the later models $V^{\mathbb{P}_{\gamma}}$ for $\gamma<\kappa$, the partial strongness of $\delta$ is weakly indestructible by all $\delta$-suitable partial orderings of rank less than $\kappa$. Note that this proof requires no use of Hamkins' Gap Forcing Theorem of [5] and [6].
} 
for any measurable cardinal $\delta \leq \sigma$. Since $\left(V_{\eta}\right)^{V^{\mathbb{P}} \sigma} \vDash$ "Any measurable cardinal $\delta>\sigma$ isn't $\delta+2$ strong", our remarks above immediately yield that $\left(V_{\eta}\right)^{V^{\mathbb{P}} \sigma} \vDash$ "Weak indestructibility for strongness holds for every measurable cardinal $\delta>\sigma$ ". Therefore, $\left(V_{\eta}\right)^{V^{\mathbb{P}} \sigma} \vDash " \sigma$ is a strong cardinal, and weak universal indestructibility for strongness holds". This completes the proof of Lemma 1.3.

If the construction of $\mathbb{P}$ does not terminate before stage $\kappa$, then the arguments of Lemma 1.3 show that weak indestructibility for strongness must hold in $V^{\mathbb{P}}$ for every measurable cardinal $\delta \neq \kappa$. The proof of Theorem 3 is consequently completed by the following lemma.

Lemma 1.4 Suppose that the definition of $\mathbb{P}$ does not terminate before stage $\kappa$. Then $V^{\mathbb{P}} \vDash " \kappa$ is a strong cardinal whose strongness is weakly indestructible".

Proof: We proceed inductively, taking as our inductive hypothesis that if $\alpha \geq 1$ is an ordinal and $N \subseteq V$ is an arbitrary transitive model of $\mathrm{ZFC}+\mathrm{GCH}$ such that $\mathbb{P} \in N, V_{\kappa} \in N$, and $N \vDash$ " $\kappa$ is $\alpha$ hyperstrong", then $N^{\mathbb{P}} \vDash$ "The $\kappa+\alpha$ strongness of $\kappa$ is weakly indestructible". For $\alpha=1$, this amounts to showing that if $\mathbb{P} \in N, V_{\kappa} \in N, N \subseteq V$, and $N \vDash$ "GCH $+\kappa$ is 1 hyperstrong", then $N^{\mathbb{P}} \vDash$ "The $\kappa+1$ strongness of $\kappa$, i.e., the measurability of $\kappa$, is weakly indestructible". To see that this is indeed the case, let $\mu \in N$ be a normal measure over $\kappa$ such that for $j_{\mu}: N \rightarrow M_{\mu}$ the ultrapower embedding via $\mu, M_{\mu} \vDash$ " $\kappa$ isn't measurable". Note that $N \vDash " \mathbb{P}=\left\langle\left\langle\mathbb{P}_{\delta}, \dot{\mathbb{Q}}_{\delta}\right\rangle: \delta<\kappa\right\rangle$ is a reverse Easton iteration such that at nontrivial stages $\delta, \Vdash_{\mathbb{P}_{\delta}}$ ' $\dot{\mathbb{Q}}_{\delta}$ is $<\delta$-strategically closed' ". The preceding two sentences yield that $j_{\mu}(\mathbb{P})=\mathbb{P} * \dot{\mathbb{Q}}^{\prime}$, where the first ordinal at which $\dot{\mathbb{Q}}^{\prime}$ is forced to do nontrivial forcing is above $\kappa^{+}$, and $\vdash_{\mathbb{P}}$ “这' is $\prec \kappa^{+}$-strategically closed". Since $N \vDash \mathrm{GCH}$, standard arguments yield that $j_{\mu}$ lifts in $N$ to $j_{\mu}: N^{\mathbb{P}} \rightarrow M_{\mu}^{j_{\mu}(\mathbb{P})}{ }^{5}$ From this, it follows that $N^{\mathbb{P}} \vDash " \kappa$ is measurable". Since the measurability of $\kappa$ is weakly indestructible, we have established the base case of our induction.

\footnotetext{
${ }^{5}$ An outline of these arguments is as follows. Let $G$ be $N$-generic over $\mathbb{P}$. Since $\mathbb{P}$ is $\kappa$-c.c., $M_{\mu}[G]$ remains $\kappa$-closed with respect to $N[G]$. Because $N \vDash \mathrm{GCH}$ and $M_{\mu}$ is given by an ultrapower embedding, we may let $\left\langle D_{\alpha}: \alpha<\kappa^{+}\right\rangle \in N[G]$ enumerate the dense open subsets of $\mathbb{Q}^{\prime}$ present in $M_{\mu}[G]$. As in the construction of the generic object $H^{\prime}$ given later in the proof of this lemma, it is possible to use the $\prec \kappa^{+}$-strategic closure of $\mathbb{Q}^{\prime}$ in both $M_{\mu}[G]$ and $N[G]$ to build in $N[G]$ an $M_{\mu}[G]$-generic object $G^{\prime}$ over $\mathbb{Q}^{\prime}$. Since $j_{\mu}^{\prime \prime} G \subseteq G * G^{\prime}, j_{\mu}$ lifts to $j_{\mu}: N[G] \rightarrow M_{\mu}[G]\left[G^{\prime}\right]$.
} 
We now assume that $\alpha>1$ is an arbitrary (successor or limit) ordinal. If our inductive hypothesis is false at $\alpha$, then let $N$ and $\mathbb{Q} \in N^{\mathbb{P}}$ which is $\kappa$-suitable be such that $N^{\mathbb{P} * \dot{\mathbb{Q}}} \vDash$ " $\kappa$ isn't $\kappa+\alpha$ strong". For the sake of simplicity, we assume without loss of generality that $N=V$. Choose $\lambda$ to be sufficiently large, e.g., suppose $\lambda$ is the least strong limit cardinal above $\max (|\operatorname{TC}(\mathbb{P} * \dot{\mathbb{Q}})|, \kappa+\alpha)$ having cofinality $\kappa$. Let $j: V \rightarrow M$ be an elementary embedding witnessing the $\lambda$ strongness of $\kappa$ generated by a $(\kappa, \lambda)$-extender such that $M \vDash " \kappa$ is $\beta$ hyperstrong for every $\beta<\alpha$ ". By the choice of $j$ and $M, \mathbb{Q} \in M^{\mathbb{P}}$. Since $V \vDash$ "No cardinal $\beta>\kappa$ is $\beta+2$ strong" and $V_{\lambda} \subseteq M, M \vDash$ "There are neither strong cardinals nor cardinals $\beta$ which are $\beta+2$ strong in either the half-open interval $(\kappa, \lambda]$ or the half-open interval $(\kappa,|\mathbb{Q}|] "$. From this, we may immediately infer that $M^{\mathbb{P}} \vDash$ "Q⿱Q has rank below the least $M$-strong cardinal above $\kappa$ and forcing with $\mathbb{Q}$ destroys the $\kappa+\alpha$ strongness of $\kappa "$.

In addition, it is the case that forcing with $\mathbb{Q}$ leaves no cardinals $\beta$ which are $\beta+2$ strong in the half-open interval $(\kappa,|\mathbb{Q}|]$. To see this, note that it is possible (in either $V$ or $M$ ) to write $\mathbb{P} * \dot{\mathbb{Q}}=\mathbb{P}^{\prime} * \dot{\mathbb{P}}^{\prime \prime}$, where $\left|\mathbb{P}^{\prime}\right|=\omega, \mathbb{P}^{\prime}$ is nontrivial, and $\vdash_{\mathbb{P}^{\prime}}$ " $\dot{\mathbb{P}}^{\prime \prime}$ is (at least) $\aleph_{1}$-strategically closed". By Hamkins' Gap Forcing Theorem of [5] and [6], this means that any cardinal $\beta \in(\kappa,|\mathbb{Q}|]$ which is $\beta+2$ strong in $M^{\mathbb{P} * \dot{\mathbb{Q}}}$ had to have been $\beta+2$ strong in $M$. As we have observed above, there can be no such cardinals. Further, by the choice of $j$ and $M$, it is inductively the case that $M^{\mathbb{P}} \vDash$ "The $\kappa+\beta$ strongness of $\kappa$ is weakly indestructible for every $\beta<\alpha "$. Consequently, $\mathbb{Q}$ is an allowable choice in the stage $\kappa$ lottery held in $M^{\mathbb{P}}$ in the definition of $j(\mathbb{P})$, so above the appropriate condition $q, j(\mathbb{P} * \dot{\mathbb{Q}})$ is forcing equivalent to $\mathbb{P} * \dot{\mathbb{Q}} * \dot{\mathbb{R}} * j(\dot{\mathbb{Q}})$, where the first nontrivial stage in $\dot{\mathbb{R}}$ is forced to occur well above $\lambda$. For the remainder of the proof of Lemma 1.4, we assume without loss of generality that we are forcing above $q$.

We now show that the embedding $j$ lifts in $V^{\mathbb{P} * \dot{\mathbb{Q}}}$ to $j: V^{\mathbb{P} * \dot{\mathbb{Q}}} \rightarrow M^{j(\mathbb{P} * \dot{\mathbb{Q}})}$. The methods for doing this are quite similar to those given in the proof of [7, Theorem 4.10] (as well as elsewhere). For the benefit of readers, we give the argument here as well, taking the liberty to quote freely from [7, Theorem 4.10]. Because $j$ is an extender embedding, we have that $M=\left\{j(f)(a): a \in[\lambda]^{<\omega}\right.$, $f \in V$, and $\left.\operatorname{dom}(f)=[\kappa]^{|a|}\right\}$. Since we may write $j(\mathbb{P})$ as $\mathbb{P} * \dot{\mathbb{Q}} * \dot{\mathbb{R}}$, as we have already observed, 
we know that the first ordinal at which $\dot{\mathbb{R}}$ is forced to do a lottery sum is above $\lambda$. Since $\lambda$ has been chosen to have cofinality $\kappa$, we may assume that $M^{\kappa} \subseteq M$. This means that if $G$ is $V$-generic over $\mathbb{P}$ and $H$ is $V[G]$-generic over $\mathbb{Q}, \mathbb{R}$ is $\prec \kappa^{+}$-strategically closed in both $V[G][H]$ and $M[G][H]$, and $\mathbb{R}$ is $\lambda$-strategically closed in $M[G][H]$.

As in [7], by using a suitable coding that allows us to identify finite subsets of $\lambda$ with elements of $\lambda$, by the definition of $M$, there must be some $\alpha_{0}<\lambda$ and function $g$ such that $\dot{\mathbb{Q}}=j(g)\left(\alpha_{0}\right)$. Let $N^{*}=\left\{i_{G * H}(\dot{z}): \dot{z}=j(f)\left(\kappa, \alpha_{0}, \lambda\right)\right.$ for some function $\left.f \in V\right\}$. It is easy to verify that $N^{*} \prec M[G][H]$, that $N^{*}$ is closed under $\kappa$ sequences in $V[G][H]$, and that $\kappa, \alpha_{0}, \lambda, \mathbb{Q}$, and $\mathbb{R}$ are all elements of $N^{*}$. Further, since $\mathbb{R}$ is $j(\kappa)$-c.c. in $M[G][H]$ and there are only $2^{\kappa}=\kappa^{+}$many functions $f:[\kappa]^{3} \rightarrow V_{\kappa}$ in $V$, there are at most $\kappa^{+}$many dense open subsets of $\mathbb{R}$ in $N^{*}$. Therefore, since $\mathbb{R}$ is $\prec \kappa^{+}$-strategically closed in both $M[G][H]$ and $V[G][H]$, we can build $H^{\prime} \subseteq \mathbb{R}$ in $V[G][H]$ as follows. Let $\left\langle D_{\sigma}: \sigma<\kappa^{+}\right\rangle$enumerate in $V[G][H]$ the dense open subsets of $\mathbb{R}$ present in $N^{*}$ so that every dense open subset of $\mathbb{R}$ occurring in $N^{*}$ appears at an odd stage at least once in the enumeration. If $\sigma$ is an odd ordinal, $\sigma=\tau+1$ for some $\tau$. Player I picks $p_{\sigma} \in D_{\sigma}$ extending $q_{\tau}$ (initially, $q_{0}$ is the empty condition), and player II responds by picking $q_{\sigma} \geq p_{\sigma}$ according to a fixed strategy $\mathcal{S}$ (so $q_{\sigma} \in D_{\sigma}$ ). If $\sigma$ is a limit ordinal, player II uses $\mathcal{S}$ to pick $q_{\sigma}$ extending each $q \in\left\langle q_{\gamma}: \gamma<\sigma\right\rangle$. By the $\prec \kappa^{+}$-strategic closure of $\mathbb{R}$ in $V[G][H]$, player II's strategy can be assumed to be a winning one, so $\left\langle q_{\sigma}: \sigma<\kappa^{+}\right\rangle$can be taken as an increasing sequence of conditions with $q_{\sigma} \in D_{\sigma}$ for $\sigma<\kappa^{+}$.

Let $H^{\prime}=\left\{p \in \mathbb{R}: \exists \sigma<\kappa^{+}\left[q_{\sigma} \geq p\right]\right\}$. We show now that $H^{\prime}$ is actually $M[G][H]$-generic over $\mathbb{R}$. If $D$ is a dense open subset of $\mathbb{R}$ in $M[G][H]$, then $D=i_{G * H}(\dot{D})$ for some name $\dot{D} \in M$. Consequently, $\dot{D}=j(f)\left(\kappa, \kappa_{1}, \ldots, \kappa_{n}\right)$ for some function $f \in V$ and $\kappa<\kappa_{1}<\cdots<\kappa_{n}<\lambda$. Let $\bar{D}$ be a name for the intersection of all $i_{G * H}\left(j(f)\left(\kappa, \alpha_{1}, \ldots, \alpha_{n}\right)\right)$, where $\kappa<\alpha_{1}<\cdots<\alpha_{n}<\lambda$ is such that $j(f)\left(\kappa, \alpha_{1}, \ldots, \alpha_{n}\right)$ yields a name for a dense open subset of $\mathbb{R}$. Since this name can be given in $M$ and $\mathbb{R}$ is $\lambda$-strategically closed in $M[G][H]$ and therefore $(\lambda, \infty)$-distributive in $M[G][H], \bar{D}$ is a name for a dense open subset of $\mathbb{R}$ which is definable without the parameters $\kappa_{1}, \ldots, \kappa_{n}$. Hence, by its definition, $i_{G * H}(\bar{D}) \in N^{*}$. Thus, since $H^{\prime}$ meets every dense open subset of $\mathbb{R}$ present in $N^{*}$, 
$H^{\prime} \cap i_{G * H}(\bar{D}) \neq \emptyset$, so since $\bar{D}$ is forced to be a subset of $\dot{D}, H^{\prime} \cap i_{G * H}(\dot{D}) \neq \emptyset$. This means $H^{\prime}$ is $M[G][H]$-generic over $\mathbb{R}$, so in $V[G][H]$, as $j^{\prime \prime} G \subseteq G * H * H^{\prime}, j$ lifts to $j: V[G] \rightarrow M[G][H]\left[H^{\prime}\right]$ via the definition $j\left(i_{G}(\tau)\right)=i_{G * H * H^{\prime}}(j(\tau))$.

It remains to lift $j$ through the forcing $\mathbb{Q}$ while working in $V[G][H]$. To do this, it suffices to show that $j^{\prime \prime} H \subseteq j(\mathbb{Q})$ generates an $M[G][H]\left[H^{\prime}\right]$-generic object $H^{\prime \prime}$ over $j(\mathbb{Q})$. Given a dense open subset $D \subseteq j(\mathbb{Q}), D \in M[G][H]\left[H^{\prime}\right], D=i_{G * H * H^{\prime}}(\dot{D})$ for some name $\dot{D}=j(\vec{D})(a)$, where $a \in[\lambda]^{<\omega}$ and $\vec{D}=\left\langle D_{\sigma}: \sigma \in[\kappa]^{|a|}\right\rangle$ is a function. We may assume that every $D_{\sigma}$ is a dense open subset of $\mathbb{Q}$. Since $\mathbb{Q}$ is $(\kappa, \infty)$-distributive, it follows that $D^{\prime}=\bigcap_{\sigma \in[\kappa]^{|a|}} D_{\sigma}$ is also a dense open subset of Q. As $j\left(D^{\prime}\right) \subseteq D$ and $H \cap D^{\prime} \neq \emptyset, j^{\prime \prime} H \cap D \neq \emptyset$. Thus, $H^{\prime \prime}=\left\{p \in j(\mathbb{Q}): \exists q \in j^{\prime \prime} H[q \geq p]\right\}$ is our desired generic object, and $j$ lifts in $V[G][H]$ to $j: V[G][H] \rightarrow M[G][H]\left[H^{\prime}\right]\left[H^{\prime \prime}\right]$. This final lifted version of $j$ is $\lambda$ strong since $V_{\lambda} \subseteq M$, meaning $\left(V_{\lambda}\right)^{V[G][H]} \subseteq M[G][H] \subseteq M[G][H]\left[H^{\prime}\right]\left[H^{\prime \prime}\right]$. Therefore, since $V[G][H] \vDash " \lambda>\kappa+\alpha$ is a strong limit cardinal", $V[G][H] \vDash " \kappa$ is $\kappa+\alpha$ strong". This contradiction completes our induction and the proof of Lemma 1.4.

Lemmas $1.2-1.4$ complete the proof of Theorem 3 .

Now that we have finished with the proof of Theorem 3, we turn our attention to the inner model portion of our argument. Specifically, we establish the following result.

Theorem 4 Con $(Z F C+$ There is a strong cardinal + Weak universal indestructibility for strongness holds $) \Longrightarrow$ Con(ZFC + There is a hyperstrong cardinal).

Proof: We argue using standard core model techniques exposited in [10] and [11]. We are done if there is an inner model with a hyperstrong cardinal, so we assume without loss of generality that this is not the case.

Suppose that $V \vDash$ "ZFC $+\kappa$ is a strong cardinal + Weak universal indestructibility for strongness holds". Let $\lambda>\kappa$ be an arbitrary ordinal which is sufficiently large, e.g., $\lambda$ is of size at least $\kappa+\omega$. We now have that if $j: V \rightarrow M$ is an elementary embedding witnessing the $\lambda$ strongness 
of $\kappa$ generated by a $(\kappa, \lambda)$-extender $\mathcal{E}$, then $M \vDash " \kappa$ is $(\kappa+2)$ strong". By elementarity, $M \vDash$ "The $(\kappa+2)$ strongness of $\kappa$ is weakly indestructible". Consequently, for $\delta>\kappa^{+}$an arbitrary cardinal and $\mathbb{P}_{\delta}=\left(\operatorname{Coll}\left(\kappa^{+}, \delta\right)\right)^{M}, M^{\mathbb{P}_{\delta}} \vDash " \kappa$ is $(\kappa+2)$ strong". Since any subset of $\delta$ may now be coded by a subset of $\kappa^{+}$, this means that there is actually an extender $\mathcal{F} \in M^{\mathbb{P}_{\delta}}$ witnessing enough strongness to show that all subsets of $\delta$ are captured in the relevant target model. By downwards absoluteness to the core model $(K)^{M^{\mathbb{P}} \delta}$, this last fact is true in $(K)^{M^{\mathbb{P}} \delta}$ as well, i.e., in $(K)^{M^{\mathbb{P}} \delta}$, there is some strongness extender $\mathcal{F}^{*}=\mathcal{F} \uparrow(K)^{M^{\mathbb{P}} \delta}$ witnessing that all subsets of $\delta$ are captured in the relevant target model. By the absoluteness of the core model under set forcing, in the core model $(K)^{M}=(K)^{M^{\mathbb{P} \delta}}, \mathcal{F}^{*}$ is a strongness extender witnessing that all subsets of $\delta$ are captured in the relevant target model. Since $\delta>\kappa$ was arbitrary, this just means that $(K)^{M} \vDash$ " $\kappa$ is a strong cardinal".

Let $K=(K)^{V}$. We show that $K \vDash " \kappa$ is 1 hyperstrong". To do this, take once again $\lambda, \mathcal{E}$, $j$, and $M$ as in the preceding paragraph. Let $\mathcal{E}^{*}=\mathcal{E} \uparrow K$, with $i: K \rightarrow N$ the $\lambda$ strongness embedding generated by $\mathcal{E}^{*}$ and $\ell: N \rightarrow(K)^{M}$ the associated factor elementary embedding whose critical point is greater than $\kappa$. It is then the case that $N \vDash " \kappa$ is a strong cardinal", since by elementarity, $N \vDash " \kappa$ is a strong cardinal" iff $(K)^{M} \vDash " \ell(\kappa)$ is a strong cardinal", i.e., iff $(K)^{M} \vDash " \kappa$ is a strong cardinal". Thus, for any $\lambda$ which is sufficiently large, there is an elementary embedding witnessing the $\lambda$ strongness of $\kappa$ in $K$ generated by a $(\kappa, \lambda)$-extender such that in the target model, $\kappa$ is a strong cardinal.

Now that we know that $K \vDash " \kappa$ is 1 hyperstrong", we are able to proceed inductively. Specifically, we assume that for $\lambda$ having been chosen to be sufficiently large, $j: V \rightarrow M$ an elementary embedding witnessing the $\lambda$ strongness of $\kappa$ generated by a $(\kappa, \lambda)$-extender $\mathcal{E}$, and $\gamma$ either a successor or limit ordinal, $(K)^{M} \vDash$ " $\kappa$ is $\alpha$ hyperstrong for every $\alpha<\gamma$ ". The proof given in the preceding paragraph, with " $\kappa$ is $\alpha$ hyperstrong for every $\alpha<\gamma$ " replacing " $\kappa$ is a strong cardinal" then shows that $K \vDash " \kappa$ is $\gamma$ hyperstrong". Since $\gamma$ was arbitrary, this completes the proof of Theorem 4 . 
The proofs of Theorems 3 and 4 complete the proof of Theorem 1.

We note that [2, Theorems 1 and 2] may be established using a supercompact cardinal having analogous properties to those given in Definition 1.1. More specifically, these theorems may be proven using a cardinal $\kappa$ in which in Definition 1.1, strong is replaced by supercompact, and instead of elementary embeddings witnessing strongness generated by extenders, we use elementary embeddings witnessing supercompactness generated by ultrafilters. In particular, models containing a supercompact cardinal which also witness universal indestructibility for supercompactness may be so constructed, as well as models containing strongly compact cardinals which witness various forms of universal indestructibility (including universal indestructibility for strong compactness). Since these theorems were originally proven using a strengthened version of Woodin cardinal in which strongness-like elementary embeddings are replaced by supercompactness-like elementary embeddings (see [2] for the exact definition), in analogy to Theorem 2 of this paper, this weakens the hypotheses used to obtain these theorems. (The work of [2] also weakened the hypotheses used to establish universal indestructibility from the original assumptions of [1].) Of course, since inner model theory for supercompactness is still in its infancy, getting an equiconsistency for universal indestructibility for either strong compactness or supercompactness relative to a model satisfying supercompactness assumptions currently remains out of reach.

We conclude by asking whether it is possible to prove a version of Theorem 1 in which the equiconsistency is for the type of indestructibility Gitik and Shelah first described in [4]. This would seem to require a version of the Gap Forcing Theorem for Prikry iterations relative to strongness, something which has yet to be established.

\section{References}

[1] A. Apter, J. D. Hamkins, "Universal Indestructibility", Kobe Journal of Mathematics 16, 1999, $119-130$. 
[2] A. Apter, G. Sargsyan, "A Reduction in Consistency Strength for Universal Indestructibility", Bulletin of the Polish Academy of Sciences (Mathematics) 55, 2007, 1-6.

[3] S. Baldwin, "Between Strong and Superstrong", Journal of Symbolic Logic 51, 1986, 547-559.

[4] M. Gitik, S. Shelah, "On Certain Indestructibility of Strong Cardinals and a Question of Hajnal", Archive for Mathematical Logic 28, 1989, 35-42.

[5] J. D. Hamkins, "Gap Forcing", Israel Journal of Mathematics 125, 2001, 237-252.

[6] J. D. Hamkins, "Gap Forcing: Generalizing the Lévy-Solovay Theorem", Bulletin of Symbolic Logic 5, 1999, 264-272.

[7] J. D. Hamkins, "The Lottery Preparation", Annals of Pure and Applied Logic 101, 2000, 103-146.

[8] A. Kanamori, The Higher Infinite, Springer-Verlag, Berlin and New York, 1994.

[9] R. Laver, "Making the Supercompactness of $\kappa$ Indestructible under $\kappa$-Directed Closed Forcing", Israel Journal of Mathematics 29, 1978, 385-388.

[10] J. Steel, The Core Model Iterability Problem, Lecture Notes in Logic 8, Springer-Verlag, Berlin and New York, 1996.

[11] M. Zeman, Inner Models and Large Cardinals, de Gruyter Series in Logic and its Applications 5, Walter de Gruyter and Co., Berlin, 2002. 